\title{
SERIOUS GAME DEVELOPMENT AND DYNAMIC TAILORING THROUGH MODEL-DRIVEN AUTHORING
}

\author{
Sofie Van Hoecke ${ }^{1}$, Gaetan Deglorie ${ }^{1}$, Koen Samyn ${ }^{2}$ \\ ${ }^{1}$ IDLab, Ghent University - imec (BELGIUM) \\ ${ }^{2} D A E$, Howest (BELGIUM)
}

\begin{abstract}
This paper presents a model-driven authoring framework and toolset that enables to produce serious games easily and quickly, at lower cost and with the active involvement of (non-IT schooled) domain experts, and therefore lowers the barriers that hinder the production of serious games. Furthermore, the toolset allows for the creation of games with an adaptive narrative that can be played several times without becoming predictable by adapting the gameplay based on the real-time input of the player or post-game feedback. Choices made by the player have an influence on the overarching narrative of the game, which in turn will be used to select appropriate narrative events for the new context. Each narrative event can focus on a different subset of the learning objectives.
\end{abstract}

The authoring framework is used within the Friendly ATTAC project, a project that helps youngsters dealing with cyberbullying issues through an innovative serious game. By using the toolset developed, it was possible for the non-technical people (subject-matter experts) to be actively involved in the development of the serious game, to make the development process much more iterative, and at the same time to shorten the overall development time. The toolbox was successfully used to design levels that are afterwards used to fully automatically create playable adaptive game scenarios.

Keywords: Serious Games, Model-Driven Engineering, Authoring Frameworks, Dynamic Tailoring.

\section{INTRODUCTION}

Serious digital games are games considered to be both instructional and fun, by being highly enjoyable, attention-captivating and intrinsically motivating [1]. Serious games can combine the advantages of computer processing, with high levels of attractiveness. They differ from mere entertainment games in their primary aim, which is non-entertainment and often related to education, training or promotion of behavior change.

The popularity of serious games has raised the need for high-level authoring environments in order to (1) allow people without professional game design skills to create (or being actively involved in the creation) or modify serious games [3], and (2) enhance serious game development, as development costs are one of the major drawbacks [2].

To come to well-grounded and effective serious games, different parties should be involved in the development of serious games, game developers as well as so-called domain experts, such as pedagogical experts or psychologists and subject-matter experts, but this may result in communication barriers that can seriously hinder the development. To solve this issue, we developed a model-driven authoring framework and toolset that enables to produce serious games easily and quickly, at lower cost and with the active involvement of (non-IT schooled) domain experts, and therefore lowers the barriers that hinder the production of serious games.

Model-driven authoring with domain experts is however not sufficient to create successful serious games. One specific method that was proven effective in serious games is individual tailoring [4] of the game challenge that is provided to players. More specifically, dynamic tailoring has been recommended to continuously adjust the difficulty level of the challenge the player can master to ensure flow and provide all players with an optimal level of challenge [5][6]. A recent meta-analysis of serious games for healthy lifestyle promotion indeed indicated that games with challenges that are difficult and do not adjust to the player's skill level, were associated with lower effectiveness compared to simple, static challenges or to adaptive difficult challenges [7]. Simple static challenges may, however, lead to boredom and demotivation after continued game play. Adaptively tailoring the challenge to what the player is able to master may thus lead to an optimal level of both learning outcomes and motivation to play the game. 
To enable individual tailoring, our toolset allows for the creation of games with an adaptive narrative that can be played several times without becoming predictable by adapting the gameplay based on the real-time input of the player or post-game feedback. Choices made by the player have an influence on the overarching narrative of the game, which in turn will be used to select appropriate narrative events for the new context. Each narrative event can focus on a different subset of the learning objectives.

The remainder of this paper is as follows. The next section presents our model-driven authoring framework. Section 3 discusses our story engine to add non-linear narrative to serious games using scenario templates and aliasing. Finally, Section 4, respectively Section 5, present a summary of the results and some conclusions.

\section{MODEL-DRIVEN AUTHORING FRAMEWORK}

Figure 1 presents the general concept of our model-driven authoring framework. Several steps have to be followed in order to generate scenarios and levels for the serious game. Firstly, social scientists have to write scenarios which incorporate their theories and hypotheses they want to test or model. Secondly, via the authoring framework they can translate their scenarios into ATTAC-L scenarios and add non-linear narrative. The ATTAC-L language [10] is a graphically illustrated modelling language for educational virtual scenarios, understandable by non-technical people. By connecting bricks, game moves, but also entire scenarios and game levels, can be unambiguously described in a humanreadable form. In parallel, they can model the visual aspects of the game levels using our sandbox [11] that offers features tailored to the development of serious games. The third step consists of the XML file generation based on the ATTAC-L scenarios on one hand and the sandbox configuration on the other hand. Finally, the fourth step takes the XML file and translates it into actual game level and moves [11].

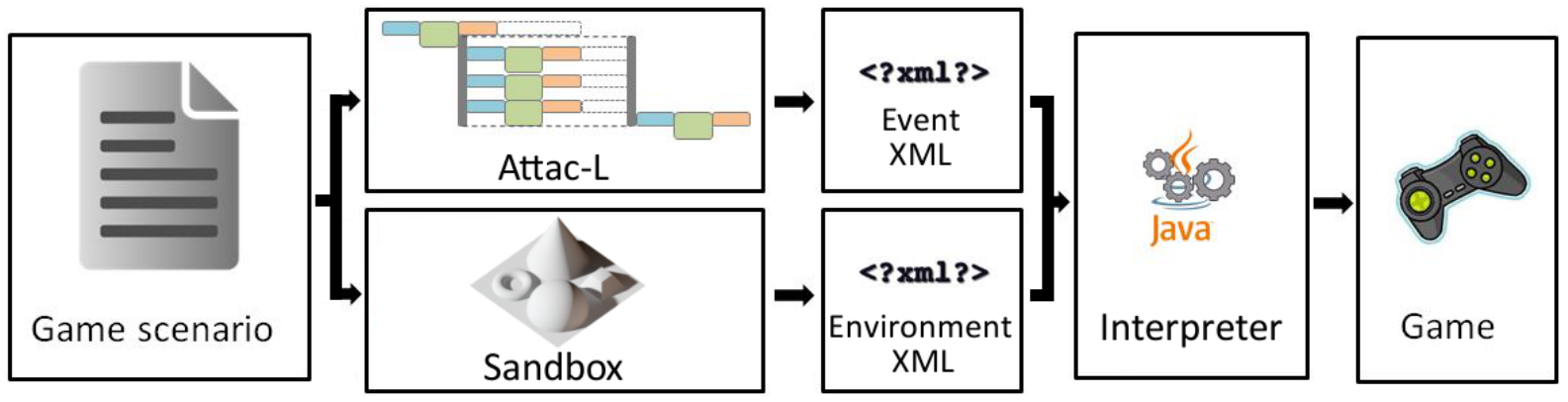

Figure 1: General concept of process to generate game scenarios and levels

The authoring framework is used within the Friendly ATTAC project, a project that helps youngsters dealing with cyberbullying issues through an innovative serious game. Cyberbullying, i.e., bullying via electronic communication tools, is a relatively recent phenomenon that especially occurs among early adolescents (12-15-year olds). The Internet offers this generation of youngsters ample opportunities to communicate with peers. However, in their social interactions on the Internet, youngsters can be confronted with undesirable situations. As cyberbullying may have a serious impact on the mental (and physical) wellbeing of the victims, many societal actors are currently involved in anticyberbullying initiatives. They argue strongly for evidence-based, appealing, ICT-related intervention tools, such as serious games, that empower youngsters dealing with cyberbullying.

By means of virtual experience scenarios, providing players with immediate feedback in a safe computer-mediated environment, the project attempts to modify behaviour patterns of bullies, bystanders and victims. This is done by allowing youngsters, using virtual scenarios, experience different roles in cyberbullying incidents, and by giving them adjusted feedback based on their individual reactions. In this way, we hope to make youngsters more aware of the consequences of certain behaviour. Bullies will be encouraged to cease bullying by increasing their empathy, victims will be taught adequate coping strategies and bystanders (witnesses of the incident) will be sensibilized to intervene.

The resulting game is a 2,5 D puzzle-adventure game in which the player has to remove cyberbullying webpages by correctly applying positive bystander strategies in a classroom context. The player's response affects the victim's mood, the classroom climate and the bully's level of bullying. Mechanics involve a discussion system in which the player needs to assess the situation, character development 
where characters can evolve to several participant roles in cyberbullying, and a reputation system where the classroom climate is set as a final game objective.

\section{ADAPTIVE NARRATIVES FOR SERIOUS GAMES}

Adaptivity and adaptation has already been extensively discussed and researched in different contexts. In the ELEKTRA project [8], micro-adaptivity is introduced to guide and support learners in acquiring knowledge. Micro-adaptivity is adaptation within learning tasks. Micro-adaptivity affects only the presentation of a learning object or a learning situation. It is achieved without compromising the learner's gaming experience. There are methods for predicting the actions that the player might take while playing the game and then adapting (aspects of) the game accordingly [9], as well as methods for generating adaptive game worlds based on the players and their experience model [9]. In the European FearNot! Project [12], episodes of bullying are observed by the player. In between these episodes, the player takes over the role of an invisible friend to the victimized character and watches how the story further develops as a result of his advices.

Although the ELEKTRA and FearNot! project recognize the need for an adaptive gameplay in serious games, examples of adaptive narratives are hard to find in the wide range of commercial and serious games. This should not come as a surprise because there are many hurdles to take. Every bifurcation in the story results in a doubling of the number of scenarios that are needed to define the game. A naive approach towards the implementation of adaptive narratives leads to a huge amount of work [13], and/or compromises that reduce the combinatorial explosion [12].

In [14], an approach based on cognitive science is presented and the authors also believe that learners/players benefit from a layered and adaptive approach to learning. This layered approach was applied for the serious game CyberCIEGE by clustering scenarios in layers with greater scenario complexity in each successive layer. However, adaptability was not applied as this issue required a longer-term research and develop vision for CyberCIEGE. We present a similar clustering approach but cluster the scenarios in terms of performance or learning objectives. The CyberCIEGE game also does not adopt a player model as we do.

In the project Muse [15], a method is presented that allows content creators to create an interactive drama by adopting the concepts of providence and the Zelig interaction metaphor, which means that non-player characters can automatically take on roles that are necessary for the advancement of the storyline. Our presented approach is similar but introduces additional conditions on the role taking process.

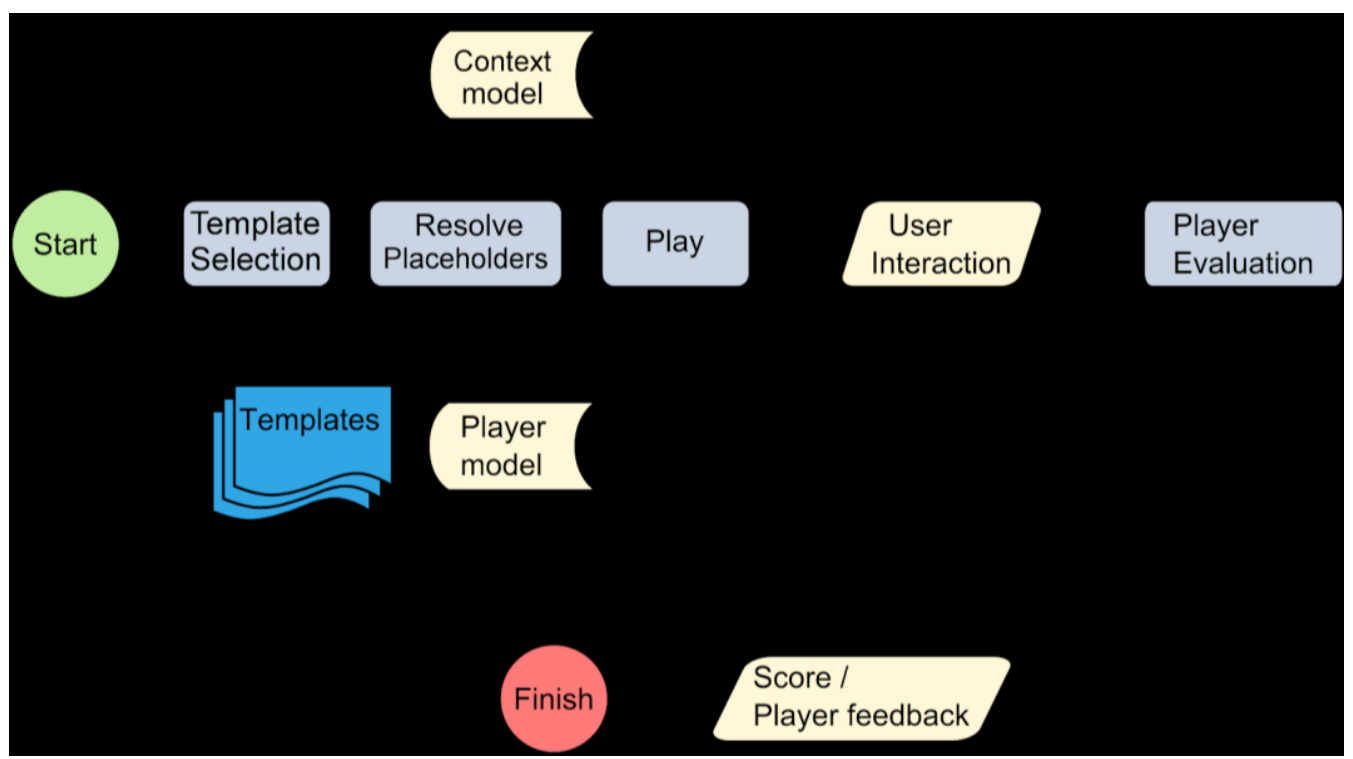

Figure 2: Overview of the inner workings of the story engine

Figure 2 presents how adaptive narratives are enabled within the authoring framework. First, a player model is created maintaining information and progress of the player towards the pedagogical objectives of the game. Second, a story engine is created that selects a scenario template from a scenario database based on the current state of the player model and provides the template with 
contextual information from the game. Therefore, a context model is derived from the environment model created in the sandbox, by querying this environment model for a list of objects and characters near the player. The model is then used to resolve the placeholders in the scenario templates. Once all placeholders have been resolved, the script is turned into a playable scenario which is executed by the interpreter (Figure 1 step 4).

This way, the number of actual possible in-game scenarios is much larger than the number of modelled scenarios. By tracking the player's learning state in real-time, the gameplay and occurrence of learning challenges can be adapted to it to better suit the problem areas of the player. During each playthrough the player is only presented with a small set of the total possible scenarios (those that match the current game context), thus promoting replayability and serving as a method to create an adaptive gameplay, i.e., adapting the gameplay based on the interaction of the player (and maintained in the player model).

Whenever the player plays a scenario, the interaction will be evaluated and the player model will be updated. For instance, for the game developed in the context of the Friendly ATTAC project, each choice presented to the player in a scenario might or might not contribute to the pedagogical objective associated with the scenario, influencing the corresponding objective weight in the player model accordingly. If the player fails an event tagged with a certain pedagogical objective, the weight of that objective is increased in the player model, leading to a higher chance of scenarios tagged with the same objective being selected.

\section{RESULTS}

By using the toolset developed, technical complexity of writing scenarios for serious games was reduced by separating the modelling scenario template language from the serious game implementation. By decoupling the modelling and implementation, a writer can develop a scenario with only an abstract knowledge of the game world, and does not need to concern him- or herself with the technical details of the game engine. This way, it was possible for the non-technical people (subjectmatter experts) to be actively involved in the development of the serious game, to make the development process much more iterative, and at the same time to shorten the overall development time. The toolbox was successfully used to design multiple levels of the Friendly ATTAC serious game that are afterwards used to fully automatically create playable adaptive game scenarios.

\section{CONCLUSIONS}

We presented a model-driven authoring framework that allows non-technical people to manipulate the $3 \mathrm{D}$ visuals, model the scenarios, and easily add non-linear narrative to the game. The different tools have been implemented and are used to build a serious game for the Friendly ATTAC project in order to help youngsters with cyberbullying.

Additionally, a story engine was introduced to add non-linear narrative to serious games using scenario templates and aliasing. The aliasing approach facilitates the design process by using simpler building blocks (scenario templates) to create a dynamic experience with an individualized storyline inside the game. Specific to the topic of serious games and learning is the creation of a player-driven narrative that tests the player on his/her skills. This was achieved through the mapping of performance objectives to each scenario template using metadata and by creating a model of a player that is adapted towards.

\section{ACKNOWLEDGEMENTS}

Friendly ATTAC (Adaptive Technological Tools Against Cyberbullying, http://www.friendlyattac.be) is an interdisciplinary research project, financed by IWT (the Flemish government agency for Innovation by Science and Technology). The main aim of the project is to investigate how ICT-related tools can be effectively used in health interventions with regard to cyberbullying amongst youngsters.

\section{REFERENCES}

[1] Prensky, M., Digital Game-Based Learning, Paragon House, 2007. 
[2] Bellotti, F., Berta, R., De Gloria, A., Designing effective serious games: opportunities and challenges for research. Int. J. Emerg. Technol. Learn. 5, 22-35, 2010.

[3] Djaouti, D., Alvarez, J., Jessel, J.P., Can gaming 2.0 help design serious games? A comparative study. Proc. of SIGGRAPH, Los Angeles, CA, USA, 2010.

[4] DeSmet, A., Van Ryckeghem, D., Compernolle, S., Baranowski, T., Thompson, D., Crombez, G., Poels, K., et al., A Meta-analysis of Serious Digital Games for Healthy Lifestyle Promotion. Preventive Medicine 69: 95-107. 2014.

[5] Charles, Darryl \& Mcneill, Michael \& Mcalister, Moira \& Black, Michaela \& Moore, Adrian \& Stringer, Karl \& Kücklich, Julian \& Kerr, Aphra. (2005). Player-centred game design: Player modelling and adaptive digital games. Proceedings of DiGRA 2005 Conference: Changing Views - Worlds in Play.

[6] Wilson, Dawn \& A Alia, Kassandra \& Kitzman, Heather \& Resnicow, Ken. (2013). A Pilot Study of the Effects of a Tailored Web-Based Intervention on Promoting Fruit and Vegetable Intake in African American Families. Childhood obesity (Print). 10. 10.1089/chi.2013.0070.

[7] DeSmet, Ann, Bastiaensens, S., Van Cleemput, K., Poels, K., Vandebosch, H., Deboutte, G., Herrewijn, L., et al. (2018). The efficacy of the friendly attac serious digital game to promote prosocial bystander behavior in cyberbullying among young adolescents : a cluster-randomized controlled trial. COMPUTERS IN HUMAN BEHAVIOR, 78, 336-347.

[8] Kickmeier-Rust, M.D., Schwarz, D., Albert, D., Verpoorten, D., Castaigne, J.-L., \& Bopp, M. (2006). The ELEKTRA project: Towards a new learning experience. In M. Pohl, A. Holzinger, R. Motschnig, \& C. Swertz (Eds.), M3 - Interdisciplinary aspects on digital media \& education (pp. 19-48). Vienna: Österreichische Computer Gesellschaft.

[9] De Troyer O., Kleinermann F., Ewais A. (2010) Enhancing Virtual Reality Learning Environments with Adaptivity: Lessons Learned. In: Leitner G., Hitz M., Holzinger A. (eds) $\mathrm{HCl}$ in Work and Learning, Life and Leisure. USAB 2010. Lecture Notes in Computer Science, vol 6389. Springer, Berlin, Heidelberg

[10] Van Broeckhoven, F., \& De Troyer, O. (2013). ATTAC-L: A modeling language for educational virtual scenarios in the context of preventing cyber bullying. In Serious Games and Applications for Health (SeGAH), 2013 IEEE 2nd International Conference on (pp. 1-8). IEEE.

[11] Van Hoecke, Sofie, Samyn, K., Deglorie, G., Janssens, O., Lambert, P., \& Van de Walle, R. (2016). Enabling control of 3D visuals, scenarios and non-linear gameplay in serious game development through model-driven authoring. In C. V. de Carvalho, P. Escudeiro, \& A. Coelho (Eds.), Lecture Notes of the Institute for Computer Sciences Social Informatics and Telecommunications Engineering (Vol. 161, pp. 103-110). Presented at the 5th International conference on Serious Games, Interaction, and Simulation (SGAMES 2015), New York, NY, USA: Springer.

[12] Vannini, Natalie, Sibylle Enz, Maria Sapouna, Dieter Wolke, Scott Watson, Sarah Woods, Kerstin Dautenhahn, Lynne Hall, Ana Paiva, Elizabeth André, Ruth Aylett, and Wolfgang Schneider. "'FearNot!": A Computer-based Anti-bullying-programme Designed to Foster Peer Intervention." European Journal of Psychology of Education 26, no. 1 (2011): 21-44.

[13] Niehaus, J., Riedl, M. Towards Scenario Adaptation for Learning, Proceedings of the 2009 conference on Artificial Intelligence in Education: Building Learning Systems that Care: From Knowledge Representation to Affective Modelling, Pages 686-688, 2009.

[14] Greitzer, Frank \& Anna Kuchar, Olga \& Huston, Kristy. (2007). Cognitive science implications for enhancing training effectiveness in a serious gaming context. ACM Journal of Educational Resources in Computing. 7. 10.1145/1281320.1281322.

[15] Llobera, J., Blom, K. J., and Slater, M. (2013). Telling stories within immersive virtual environments. Leonardo, 46(5):471-476. 